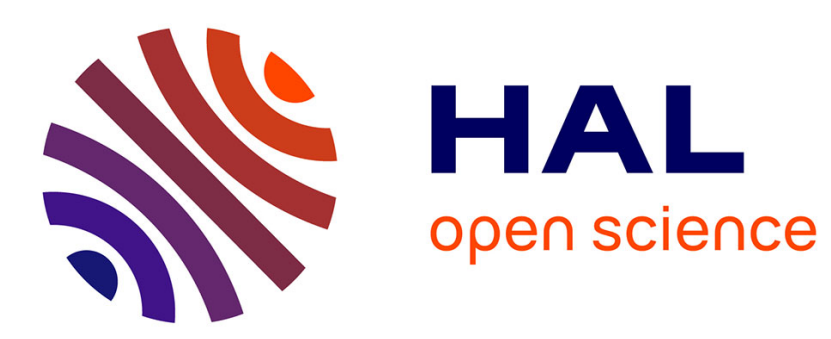

\title{
Ellipsoidal invariant sets for saturated hybrid systems
}

Mirko Fiacchini, Sophie Tarbouriech, Christophe Prieur

\section{To cite this version:}

Mirko Fiacchini, Sophie Tarbouriech, Christophe Prieur. Ellipsoidal invariant sets for saturated hybrid systems. ACC 2011 - American Control Conference, Jun 2011, San Francisco, Californie, United States. 7p. hal-00560880

\section{HAL Id: hal-00560880 \\ https://hal.science/hal-00560880}

Submitted on 30 May 2011

HAL is a multi-disciplinary open access archive for the deposit and dissemination of scientific research documents, whether they are published or not. The documents may come from teaching and research institutions in France or abroad, or from public or private research centers.
L'archive ouverte pluridisciplinaire HAL, est destinée au dépôt et à la diffusion de documents scientifiques de niveau recherche, publiés ou non, émanant des établissements d'enseignement et de recherche français ou étrangers, des laboratoires publics ou privés. 


\title{
Ellipsoidal invariant sets for saturated hybrid systems
}

\author{
Mirko Fiacchini, Sophie Tarbouriech and Christophe Prieur
}

\begin{abstract}
The paper addresses the problem of characterizing and computing an estimation of the basin of attraction for saturated hybrid systems. Hybrid systems presenting saturation on signals involved in both the continuous-time and the discretetime dynamics are considered. A geometrical characterization of local convergence is provided and employed to extend results proper of continuous-time and discrete-time saturated systems to the hybrid ones. A computation oriented condition of local convergence is given in form of convex constraints.
\end{abstract}

\section{INTRODUCTION}

Hybrid systems are dynamical systems in which both continuous-time and discrete-time dynamics are present. The growing importance of hybrid systems is mainly due to the increasing application of digital devices for the control of real systems, as chemical processes, communication and automotive systems, among others. The main issue is that the classical control theories, concerning continuous-time and discrete-time systems, do not apply to many of the problems raised in this context. Proper analysis and control techniques have to be investigated and developed, see for instance [4], [5], [8], [11], [16], and [13], [18] concerning reset systems.

In this paper we consider the problem of computing ellipsoidal estimations of the basin of attraction for saturated hybrid systems. Ellipsoidal estimates, although on one hand entail a certain degree of conservativeness (with respect to polytopes for instance), permits to pose the problem in an efficiently solvable way. Notice that the problem is tightly linked to Lyapunov theory for quadratic functions, and can be posed as a convex optimization one, in certain cases. The issue of computation of ellipsoidal estimations of the basin of attraction for linear saturated systems, both in continuoustime and discrete-time, has been dealt with in [2], [6], [9], [10]. Also polytopic estimations, for linear saturated systems and similar, have been considered, see [1], [3].

A first contribution of our paper is the geometrical characterization of contractiveness of ellipsoids using convexity related properties. First we prove that the value of a linear saturated function is contained in a set determined by a finite number of vertices, easily computable. Such result permits to characterize the Lyapunov condition for quadratic functions by means of convex constraints. The condition is useful from computational point but it also provides a deeper

This work was partially supported by the ANR project ArHyCo, ARPEGE, contract number ANR-2008 SEGI 004 01-30011459.

M. Fiacchini and S. Tarbouriech are with CNRS; LAAS; 7 avenue du colonel Roche, F-31077 Toulouse, France, Université de Toulouse; UPS, INSA, INP, ISAE; LAAS; F-31077 Toulouse, France. \{fiacchini, sophie.tarbouriech\} @laas.fr.

C. Prieur is with Department of Automatic Control, Gipsa-lab, Domaine universitaire, 961 rue de la Houille Blanche, BP 46, 38402 Grenoble, France. christophe.prieur@gipsa-lab.grenoble-inp.fr. theoretical insight to the problem of local convergence of saturated systems. Some of the results present in literature for continuous-time and discrete-time linear saturated systems are improved or recovered as particular cases of our approach. The extension of such results to obtain a condition for local convergence also for saturated hybrid systems is another contribution of the paper. Finally, a computation oriented condition for a function to be a local quadratic Lyapunov function is stated and applied to reset systems.

The paper is organized as follows: Section II presents the problem statement. Section III provides the geometrical characterization of local convergence for saturated hybrid systems. In Section IV some computation oriented considerations are given and in Section $\mathrm{V}$ the presented method is applied to the model of a reset system. The paper ends with a section of conclusions.

\section{Notation}

Given $n \in \mathbb{N}$, define $\mathbb{N}_{n}=\{x \in \mathbb{N}: 1 \leq x \leq n\}$. Given $A \in \mathbb{R}^{n \times m}, A_{i}$ with $i \in \mathbb{N}_{n}$ denotes its $i$-th row, $A_{(j)}$ with $j \in \mathbb{N}_{m}$ its $j$-th column. For two symmetric matrices, $A$ and $B, A>B$ means that $A-B$ is positive definite. The identity matrix of order $n$ is denoted $I_{n}$, the null $m \times n$ matrix is denoted $0_{m \times n}$. Operators $\leq, \geq,<$ and $>$ are intended to apply element-wise to vectors $a, b \in \mathbb{R}^{n}$. Given the symmetric positive definite matrix $P \in \mathbb{R}^{n \times n}$, define the ellipsoid $\mathscr{E}(P)=$ $\left\{x \in \mathbb{R}^{n}: x^{T} P x \leq 1\right\}$. Given the set $D$ and $\alpha \geq 0$, denote $\alpha D=\{\alpha x: x \in D\}$ and $\operatorname{co}\{D\}$ is its convex hull. Given the finite set $J \subseteq \mathbb{N}_{m}$, we denote $\bar{J}=\mathbb{N}_{m}-J$, with $m \in \mathbb{N}$.

\section{Problem Statement}

Consider the following plant:

$$
\left\{\begin{array}{l}
\dot{x}_{p}(t)=A_{p} x_{p}(t)+B_{p} u_{p}(t), \\
y_{p}(t)=C_{p} x_{p}(t),
\end{array}\right.
$$

where $x_{p} \in \mathbb{R}^{n_{p}}$ is the state vector, $y_{p} \in \mathbb{R}^{p}$ is the measured output of the plant and $u_{p} \in \mathbb{R}^{m}$ is the input of the plant.

Associated to system (1), we consider a reset controller whose state is $x_{c} \in \mathbb{R}^{n_{c}}$. By using the hybrid framework introduced in [5], two regions $\mathscr{F}$ and $\mathscr{J}$ are defined and referred to as the flow and jump sets, respectively. Possible forms of $\mathscr{F}$ and $\mathscr{J}$ are presented in the following section. The reset controller is described by continuous-time dynamics if the states belong to region $\mathscr{F} \subseteq \mathbb{R}^{n_{p}+n_{c}}$ or the time variable $\tau \in \mathbb{R}$ is smaller than or equal to the parameter $\rho \in \mathbb{R}$, and it has a discrete-time behavior otherwise. That is

$$
\left\{\begin{array}{l}
\dot{x}_{c}(t, j)=A_{c} x_{c}(t, j)+B_{c} u_{c}(t), \\
y_{c}(t, j)=C_{c} x_{c}(t, j)+D_{c} u_{c}(t), \\
\dot{\tau}(t, j)=1,
\end{array}\right.
$$


if $\left(x_{p}, x_{c}\right) \in \mathscr{F}$ or $\tau \leq \rho$, and

$$
\left\{\begin{array}{l}
x_{c}(t, j+1)=A_{d} x_{c}(t, j)+B_{d} u_{d}(t) \\
\tau(t, j+1)=0
\end{array}\right.
$$

if $\left(x_{p}, x_{c}\right) \in \mathscr{J}$ and $\tau \geq \rho$, where $x_{c}(t, j) \in \mathbb{R}^{n_{c}}$ is the state of the controller at time $t$ with $j$ occurred resets before time $t, x_{c}(t, j)$ is the state value before a jump, $x_{c}(t, j+1)$ after a jump and $y_{c} \in \mathbb{R}^{m}$ is the controller output. Variables $u_{c}(t) \in$ $\mathbb{R}^{p}$ and $u_{d}(t) \in \mathbb{R}^{n_{c}}$ are the inputs to the continuous-time and the discrete-time dynamics of the controller, respectively.

The variable $\tau$ corresponds to the so-called "temporal regularization", that permits to impose the condition that successive jumps are not allowed before a certain time interval of length $\rho>0$. The objective of the temporal regularization is to avoid an infinite number of jumps in a finite interval of time, i.e. to avoid Zeno solutions [5].

We suppose that magnitude limitations on the input of the plant are present. Also a bound on the amplitude of the discrete-time dynamics variation is assumed. That is, we pose

$$
u_{p}=\varphi\left(y_{c}\right), \quad u_{d}=\varphi\left(x_{c}\right),
$$

where $\varphi: \mathbb{R}^{a} \rightarrow \mathbb{R}^{a}$, with $a \in \mathbb{N}$, is the saturation function

$$
\varphi_{i}(y)=\operatorname{sgn}\left(y_{i}\right) \min \left\{\left|y_{i}\right|, 1\right\},
$$

for every $i \in \mathbb{N}_{a}$, for all $y \in \mathbb{R}^{a}$. The controller input is the plant output, that is $u_{c}=y_{p}$.

Remark 1: It is worth recalling that posing the bounds on the saturated signals $y_{i}$ to 1 in spite of through a positive vector $\bar{y} \in \mathbb{R}^{m}$, as used in some cases in literature, does not induce any loss of generality. Notice in fact that $\operatorname{sgn}\left(y_{i}\right) \min \left\{\left|y_{i}\right|, \bar{y}_{i}\right\}=\bar{y}_{i} \operatorname{sgn}\left(y_{i}\right) \min \left\{\left|y_{i} / \bar{y}_{i}\right|, 1\right\}$ for all $i \in$ $\mathbb{N}_{m}$. Then, for instance, by properly scaling matrices $B_{p}, C_{c}$ and $D_{c}$, saturations on $u_{p}$ can be expressed as in (5).

The continuous-time controller (2) is supposed to stabilize system (1), in absence of the saturation on $u_{p}$, i.e. if $u_{p}=y_{c}$.

Remark 2: The case under analysis is more general than the classical reset systems framework, in which no saturation is taken into account and the discrete-time dynamics consists essentially in setting the state of the controller to the value of 0 . Such case is easily recovered by posing $A_{d}=0_{n_{c} \times n_{c}}$, $B_{d}=0_{n_{c} \times m_{d}}$ and removing the saturation constraints.

The objective of the paper is summarized below.

Problem 1: Given the flow and jump sets, $\mathscr{F}$ and $\mathscr{J}$, determine the greatest ellipsoid $\Omega=\mathscr{E}(P)$, with $P=P^{T}>0$ such that, for any $x(0) \in \Omega$ and $\tau(0)=0$, the asymptotic stability of the system (1) in closed-loop with the controller (2) and (3) is guaranteed, with saturations as in (4).

Then, the aim is to optimize the size of the ellipsoidal estimation of the basin of attraction for the closed-loop system (1), (2) and (3) with saturations as in (4). As the framework taken into account is more general than the classical reset systems, the solution would apply to many cases of saturated hybrid systems.

\section{CONTRACTIVENESS CONDITION FOR HYBRID} SYSTEMS

The closed-loop saturated hybrid system, obtained from (1), (2) and (3), has continuous-time dynamics given by

$$
\left\{\begin{array}{l}
\dot{x}_{p}(t)=A_{p} x_{p}(t)+B_{p} \varphi\left(C_{c} x_{c}(t, j)+D_{c} C_{p} x_{p}(t)\right) \\
\dot{x}_{c}(t, j)=A_{c} x_{c}(t, j)+B_{c} C_{p} x_{p}(t) \\
\dot{\tau}(t, j)=1 \\
y_{p}(t)=C_{p} x_{p}(t) \\
y_{c}(t, j)=C_{c} x_{c}(t, j)+D_{c} C_{p} x_{p}(t)
\end{array}\right.
$$

which is valid in the flowing mode, that is when $\left(x_{p}, x_{c}\right) \in \mathscr{F}$ or $\tau \leq \rho$, and a discrete-time behavior, given by

$$
\left\{\begin{array}{l}
x_{c}(t, j+1)=A_{d} x_{c}(t, j)+B_{d} \varphi\left(x_{c}(t, j)\right), \\
\tau(t, j+1)=0,
\end{array}\right.
$$

when the jump conditions $\left(x_{p}, x_{c}\right) \in \mathscr{J}$ and $\tau \geq \rho$ are verified. Denoting the augmented state vector as $x=\left[x_{p}^{T}, x_{c}^{T}\right]^{T}$, with $x \in \mathbb{R}^{n}$ where $n=n_{p}+n_{c}$, the system is then given by

$$
\begin{cases}\dot{x}=\hat{g}(x)=\hat{A} x+\hat{B} \varphi(\hat{K} x), & \text { if } x \in \mathscr{F} \text { or } \tau \leq \rho, \\ x^{+}=\tilde{g}(x)=\tilde{A} x+\tilde{B} \varphi(\tilde{K} x), & \text { if } x \in \mathscr{J} \text { and } \tau \geq \rho,\end{cases}
$$

where we used $\dot{x}$ for $\dot{x}(t)$ and $x^{+}$for $x(t, j+1)$, and with

$$
\begin{gathered}
\hat{A}=\left[\begin{array}{cc}
A_{p} & 0_{n_{p} \times n_{c}} \\
B_{c} C_{p} & A_{c}
\end{array}\right], \hat{B}=\left[\begin{array}{c}
B_{p} \\
0_{n_{c} \times m}
\end{array}\right], \\
\tilde{A}=\left[\begin{array}{cc}
I_{n_{p}} & 0_{n_{p} \times n_{c}} \\
0_{n_{c} \times n_{p}} & A_{d}
\end{array}\right], \tilde{B}=\left[\begin{array}{c}
0_{n_{p} \times n_{c}} \\
B_{d}
\end{array}\right], \\
\hat{K}=\left[\begin{array}{ll}
D_{c} C_{p} & C_{c}
\end{array}\right], \quad \tilde{K}=\left[\begin{array}{ll}
0_{n_{c} \times n_{p}} & I_{n_{c}}
\end{array}\right] .
\end{gathered}
$$

The dynamics of $\tau$ has not been taken into account in the model, for simplicity.

Remark 3: The presence of a further saturation, on the plant output $y_{p}$, could be considered. This would entail the presence of nested saturations in the first equation of (6). The feeling of the authors is that such generalization can be addressed as in [17], and then the results of the paper can be extended to a wider class of hybrid systems.

The flow and jump sets are assumed to be defined as

$$
\begin{aligned}
& \mathscr{F}=\left\{x \in \mathbb{R}^{n}: x^{T} M x \geq 0\right\}, \\
& \mathscr{J}=\left\{x \in \mathbb{R}^{n}: x^{T} M x \leq 0\right\},
\end{aligned}
$$

where $M=M^{T} \in \mathbb{R}^{n \times n}$, as in [8]. Different kinds of flow and jump regions can be defined by (9). Consider for instance the classical reset rule (see [18], [12]). For reset systems, the output of the plant and the output of the controller are assumed one-dimensional, i.e. $p=m=1$, and the jump depends on the sign of their product. Then, in this case, $\mathscr{F}$ and $\mathscr{J}$ are given by (9) with $M=C^{T} T C$ where

$$
T=\left[\begin{array}{cc}
0 & -1 \\
-1 & 0
\end{array}\right] \in \mathbb{R}^{2 \times 2}, \quad C=\left[\begin{array}{cc}
C_{p} & 0_{1 \times n_{c}} \\
D_{c} C_{p} & C_{c}
\end{array}\right] \in \mathbb{R}^{2 \times n} .
$$

Please notice that from closure of $\mathscr{F}$ and $\mathscr{J}$, their intersection is non-empty. Considerations on this fact and its relations with non-uniqueness of solutions and robustness can be found in [5], [18]. Furthermore, the formulation (9) permits to define ellipsoidal jump region with the flow one that is its complementary on $\mathbb{R}^{n}$ (or vice versa). It is sufficient to choose symmetric positive (or negative) definite matrix $M$. 


\section{A. Inclusion condition for saturated functions}

In this section we show, first, that the image of the state $x \in \mathbb{R}^{n}$ through a saturated function $g(x)$ is contained in a set explicitly obtainable. This result, which represents one of the contribution of the paper, permits to characterize geometrically the condition of contractiveness of a set. Besides of its practical importance, the presented approach provides a novel geometrical insight and a deeper comprehension to the problem of convergence for saturated hybrid systems, as well as for continuous-time and discrete-time systems.

We introduce the support function of a set, a useful tool for dealing with convex closed sets and set inclusion relations.

Definition 1: Given a set $D \subseteq \mathbb{R}^{n}$, the support function of $D$ evaluated at $\eta \in \mathbb{R}^{n}$ is

$$
\phi_{D}(\eta)=\sup _{x \in D} \eta^{T} x
$$

The support function of a set $D$ evaluated at $\eta$ can be seen as the signed "distance" of the point of $D$ (or its closure) further from the origin, along the direction $\eta$. Set inclusion conditions can be given in terms of linear inequalities involving the support functions as recalled here (see [14], [15] for properties of support functions).

Property 1: Given two closed, convex sets $D \subseteq \mathbb{R}^{n}$ and $E \subseteq \mathbb{R}^{n}$, then $x \in D$ if and only if

$$
\eta^{T} x \leq \phi_{D}(\eta), \quad \forall \eta \in \mathbb{R}^{n}
$$

and $E \subseteq D$ if and only if

$$
\phi_{E}(\eta) \leq \phi_{D}(\eta), \quad \forall \eta \in \mathbb{R}^{n}
$$

The following theorem is enunciated for general linear functions with saturation, that is, for $g(x)=A x+B \varphi(K x)$, with adequate matrices $A, B$ and $K$. The theorem will be employed to prove results for both the continuous-time and the discrete-time dynamics, and then applied to hybrid systems. Recall that, given $J \subseteq \mathbb{N}_{m}$, we denote $\bar{J}=\mathbb{N}_{m}-J$.

Theorem 1: Given a function $g(x)=A x+B \varphi(K x)$ and the ellipsoid $\Omega=\mathscr{E}(P)$, with $P \in \mathbb{R}^{n \times n}$ symmetric positive definite, and $H(i, J) \in \mathbb{R}^{1 \times n}$ such that $|H(i, J) x| \leq 1$ for all $x \in \Omega$, for every $J \subseteq \mathbb{N}_{m}$ and every $i \in J$, then

$$
g(x) \in G(x),
$$

where $G(x)=\operatorname{co}\left\{N(J) x \in \mathbb{R}^{n}: J \subseteq \mathbb{N}_{m}\right\}$ and

$$
N(J)=A+\sum_{i \in \bar{J}} B_{(i)} K_{i}+\sum_{i \in J} B_{(i)} H(i, J),
$$

for all $J \subseteq \mathbb{N}_{m}$.

Proof: Consider $x \in \Omega$. First notice that, given $J \subseteq \mathbb{N}_{m}$ and $i \in J$, we have that $|H(i, J) x| \leq 1$ implies that $\varphi_{i}(x) \in$ $\operatorname{co}\left\{K_{i} x, H(i, J) x\right\}$. In fact, supposing that $K_{i} x \geq 0$, (case $K_{i} x<0$ is analogous), if $K_{i} x \leq 1$ then, trivially, $\varphi_{i}(K x)=K_{i} x$. If $K_{i} x>1$ then $H(i, J) x \leq 1=\varphi_{i}(K x)<K_{i} x$ and the inclusion is satisfied. This implies, for every $\eta \in \mathbb{R}^{n}$, that

$$
\eta^{T} B_{(i)} \varphi_{i}(K x) \in \operatorname{co}\left\{\eta^{T} B_{(i)} K_{i} x, \eta^{T} B_{(i)} H(i, J) x\right\} \subseteq \mathbb{R},
$$

holds for every $J \subseteq \mathbb{N}_{m}$ and every $i \in J$. This means that, for every $\eta \in \mathbb{R}^{n}$, either we have that

$$
\eta^{T} B_{(i)} K_{i} x \leq \eta^{T} B_{(i)} \varphi_{i}(K x) \leq \eta^{T} B_{(i)} H(i, J) x,
$$

or

$$
\eta^{T} B_{(i)} H(i, J) x \leq \eta^{T} B_{(i)} \varphi_{i}(K x) \leq \eta^{T} B_{(i)} K_{i} x,
$$

is satisfied. Hence for any $\eta \in \mathbb{R}^{n}$, every $J \subseteq \mathbb{N}_{m}$ and every $i \in J$ and accurate choice between the values $\eta^{T} B_{(i)} H(i, J) x$ and $\eta^{T} B_{(i)} K_{i} x$ provides an upper bound of $\eta^{T} B_{(i)} \varphi_{i}(K x)$. Thus, given $\eta \in \mathbb{R}^{n}$ and $x \in \Omega$, there exists $J(x, \eta) \subseteq \mathbb{N}_{m}$ such that the condition

$$
\begin{aligned}
& \eta^{T} A x+\eta^{T} B \varphi(K x)=\eta^{T} A x+\sum_{i \in \mathbb{N}_{m}} \eta^{T} B_{(i)} \varphi_{i}(K x) \leq \\
& \leq \eta^{T} A x+\sum_{i \in \bar{J}(x, \eta)} \eta^{T} B_{(i)} K_{i} x+\sum_{i \in J(x, \eta)} \eta^{T} B_{(i)} H(i, J(x, \eta)) x
\end{aligned}
$$

holds. Then, for any $\eta \in \mathbb{R}^{n}$, there exists $J(x, \eta) \subseteq \mathbb{N}_{m}$ such that the support function of $N(J(x, \eta)) x \in G(x)$ is greater than or equal to the support function of $g(x)$ and hence

$$
\eta^{T} g(x) \leq \phi_{G(x)}(\eta)
$$

From Property 1, the result (10) follows.

Thus, for every state $x \in \mathbb{R}^{n}$ and every saturated function $g(x)$, the image $g(x)$ is bounded by the convex set $G(x)$, whose vertices are known.

\section{B. Continuous-time contractiveness}

The application of the result provided in Theorem 1 to the case of continuous-time systems leads to a condition for an ellipsoid to be an estimation of the basin of attraction of the saturated system. It is worth noticing that the obtained result is the same as the one provided in [2], which, in turn, generalizes the condition presented in [9].

Proposition 1: Consider the continuous-time dynamics $\hat{g}(x)=\hat{A} x+\hat{B} \varphi(\hat{K} x)$ in (7). Given the ellipsoid $\Omega=\mathscr{E}(P)$, with $P \in \mathbb{R}^{n \times n}$ symmetric positive definite, the symmetric positive definite matrix $Q \in \mathbb{R}^{n \times n}$ and $\hat{H}(i, J) \in \mathbb{R}^{1 \times n}$ such that $|\hat{H}(i, J) x| \leq 1$ for all $x \in \Omega$, for every $J \subseteq \mathbb{N}_{m}$ and every $i \in J$, if

$$
\hat{N}(J)^{T} P+P \hat{N}(J) \leq-Q
$$

for all $J \subseteq \mathbb{N}_{m}$, with

$$
\hat{N}(J)=\hat{A}+\sum_{i \in \bar{J}} \hat{B}_{(i)} K_{i}+\sum_{i \in J} \hat{B}_{(i)} \hat{H}(i, J),
$$

then $\Omega$ is an ellipsoidal estimation of the basin of attraction and function $V(x)=x^{T} P x$ is a local Lyapunov function defined in $\Omega$ for the continuous-time system in (7).

Proof: Since $V(x)$ is a positive definite function and $\dot{V}(x)=\hat{g}(x)^{T} P x+x^{T} P \hat{g}(x)$, we have to prove that satisfaction of (13) for all $J \subseteq \mathbb{N}_{m}$, with $\hat{N}(J)$ in (14), implies

$$
\hat{g}(x)^{T} P x+x^{T} P \hat{g}(x) \leq-x^{T} Q x,
$$

for every $x \in \Omega$. From Theorem 1 we have that $\hat{g}(x) \in$ $\hat{G}(x)$, with $\hat{G}(x)=\operatorname{co}\left\{\hat{N}(J) x \in \mathbb{R}^{n}: J \subseteq \mathbb{N}_{m}\right\}$, which implies the existence of $J(x, \eta) \subseteq \mathbb{N}_{m}$, for any $\eta \in \mathbb{R}^{n}$, such that $\eta^{T} \hat{g}(x) \leq \eta^{T} \hat{N}(J(x, \eta)) x$, as proved for Theorem 1, see (12). Posing $\eta^{T}=x^{T} P$ and from (13), condition (15) follows and the result is proved.

Standard matrices manipulations can be used to pose the condition provided in Proposition 1 in LMI terms, as in [2]. 


\section{Discrete-time contractiveness}

As for the case of continuous-time saturated systems, Theorem 1 yields to a condition for local convergence in the context of discrete-time systems. In this case, the results presented in literature, see [10], are a particular case, more conservative, of the result presented here.

Proposition 2: Consider the discrete-time dynamics $\tilde{g}(x)=\tilde{A} x+\tilde{B} \varphi(\tilde{K} x)$ in (7). Given the ellipsoid $\Omega=\mathscr{E}(P)$, with $P \in \mathbb{R}^{n \times n}$ symmetric positive definite, the symmetric positive definite matrix $Q \in \mathbb{R}^{n}$ and $\tilde{H}(i, J) \in \mathbb{R}^{1 \times n}$ such that $|\tilde{H}(i, J) x| \leq 1$ for all $x \in \Omega$, for every $J \subseteq \mathbb{N}_{n_{c}}$ and every $i \in J$, if

$$
\tilde{N}(J)^{T} P \tilde{N}(J)-P \leq-Q,
$$

for all $J \subseteq \mathbb{N}_{n_{c}}$, with

$$
\tilde{N}(J)=\tilde{A}+\sum_{i \in \tilde{J}} \tilde{B}_{(i)} \tilde{K}_{i}+\sum_{i \in J} \tilde{B}_{(i)} \tilde{H}(i, J),
$$

then $\Omega$ is an ellipsoidal estimation of the basin of attraction and function $V(x)=x^{T} P x$ is a local Lyapunov function defined in $\Omega$ for the discrete-time system in (7).

Proof: The positive definite function $V(x)$ is such that $V\left(x^{+}\right)-V(x)=\tilde{g}(x)^{T} P \tilde{g}(x)-x^{T} P x$ for the discrete-time system in (7). Proving that fulfillment of (16) for every $J \subseteq \mathbb{N}_{n_{c}}$ implies

$$
\tilde{g}(x)^{T} P \tilde{g}(x)-x^{T} P x \leq-x^{T} Q x,
$$

for every $x \in \Omega$, leads to the result stated in the proposition. From Theorem 1 we have that $\tilde{g}(x) \in \tilde{G}(x)$, with $\tilde{G}(x)=$ $\operatorname{co}\left\{\tilde{N}(J) x \in \mathbb{R}^{n}: J \subseteq \mathbb{N}_{n_{c}}\right\}$. The quadratic function $V(x)=$ $x^{T} P x$, is convex and bounded over $\mathbb{R}^{n}$. Since the supremum of a convex function relative to a convex, compact set $C$ is attained at some extreme of $C$ (if attained at all), see [14], then there exists an extreme of set $\tilde{G}(x)$ where the maximum of $V(x)$ is achieved. Thus, there exists $J(x) \subseteq \mathbb{N}_{n_{c}}$ such that

$$
\tilde{g}(x)^{T} P \tilde{g}(x) \leq x^{T} \tilde{N}(J(x))^{T} P \tilde{N}(J(x)) x=\sup _{y \in \tilde{G}(x)} V(y) .
$$

From this and (16), inequality (18) follows.

Also in this case, an LMI formulation of the condition provided in Proposition 2 can be obtained.

\section{Hybrid systems contractiveness}

The results presented in Propositions 1 and 2, deriving from Theorem 1, are employed to state the desired condition for local convergence for saturated hybrid systems. Given an ellipsoid $\Omega=\mathscr{E}(P)$, we impose that every set $\alpha \Omega$ with $\alpha \in[0,1]$, is contractive for the continuous-time dynamics, for all $x$ contained in the flow and in the jump set. This is necessary since there is not certainty, in general, that the state after a jump is contained in the flow region. Moreover, we pose conditions which ensure that the variation of the candidate local Lyapunov function $V(x)=x^{T} P x$ during a jump plus the variation during a flowing interval of $\rho$, is negative. This, with the temporal regularization, would imply that the value of $V(x)$ must be decreasing between two successive jumps. Such condition is less conservative than imposing the condition, more intuitive, of decreasing of $V(x)$ during the jump. In fact, the increasing of $V(x)$ during a jump is allowed provided that during the successive time interval of length $\rho, V(x)$ would decrease more.

Theorem 2: Consider the hybrid system in (7). Given the ellipsoid $\Omega=\mathscr{E}(P)$, with $P \in \mathbb{R}^{n \times n}$ symmetric positive definite, $\hat{H}(i, I) \in \mathbb{R}^{1 \times n}$ and $\tilde{H}(j, J) \in \mathbb{R}^{1 \times n}$ such that $|\hat{H}(i, I) x| \leq$ 1 and $|\tilde{H}(j, J) x| \leq 1$, for all $x \in \Omega$, for every $I \subseteq \mathbb{N}_{m}$ and $i \in I$, every $J \subseteq \mathbb{N}_{n_{c}}$ and $j \in J, \lambda>0$ and $\sigma \geq 0$, if

$$
\hat{N}(I)^{T} P+P \hat{N}(I) \leq-2 \lambda P,
$$

for all $I \subseteq \mathbb{N}_{m}$, with $\hat{N}(I)$ as in (14), and

$$
\tilde{N}(J)^{T} e^{-\lambda \rho I_{n}} P e^{-\lambda \rho I_{n}} \tilde{N}(J)-\sigma M \leq P,
$$

for all $J \subseteq \mathbb{N}_{n_{c}}$, with $\tilde{N}(J)$ as in (17), then $\Omega$ is an ellipsoidal estimation of the basin of attraction and a local Lyapunov function defined in $\Omega$ for the hybrid system in (7) can be determined.

Proof: First, notice that, from temporal regularization, the system can be flowing also in the jump region. Condition (20) implies that the function $V(x)$ decreases along the continuous-time trajectories, within the whole set $\Omega$, from Proposition 1. In particular, $V(x) \geq 0$ for all $x \in \mathbb{R}^{n}$ and

$$
\dot{V}(x)=\frac{\partial V(x)}{\partial x} \dot{x}=x^{T} P \dot{x}+\dot{x}^{T} P x \leq-x^{T} \lambda P x-x^{T} P \lambda x,
$$

from Proposition 1, with $\dot{x}=\hat{g}(x)$ as defined in (7), see (15) with $Q=2 \lambda P$. Consider the system $\dot{\bar{x}}=-\lambda \bar{x}$, whose trajectories are given by $\bar{x}(t)=e^{-\lambda t I_{n}} \bar{x}(0)$. The time-derivative of function $V(x)$ along its trajectories is given by

$$
\dot{\bar{V}}(\bar{x})=\frac{\partial V(\bar{x})}{\partial \bar{x}} \dot{\bar{x}}=\bar{x}^{T} P \dot{\bar{x}}+\dot{\bar{x}}^{T} P \bar{x}=-\bar{x}^{T} \lambda P \bar{x}-\bar{x}^{T} P \lambda \bar{x},
$$

for every $\bar{x} \in \mathbb{R}^{n}$. Then, for every $x=\bar{x} \in \Omega$, the timederivatives along the trajectories of systems (7) and $\dot{\bar{x}}=-\lambda \bar{x}$ are such that $\dot{V}(x)-\dot{\bar{V}}(x) \leq 0$. Since the integral of a nonpositive function is smaller than or equal to 0 we have

$$
\begin{aligned}
& V(x(\tau))-V(x(0))=\int_{0}^{\tau} \dot{V}(x(t)) d t \leq \int_{0}^{\tau} \dot{\bar{V}}(\bar{x}) d t= \\
& =\int_{0}^{\tau} \dot{\bar{x}}^{T} P \bar{x} d t+\int_{0}^{\tau} \bar{x}^{T} P \dot{\bar{x}} d t=\bar{x}(\tau)^{T} P \bar{x}(\tau)-\bar{x}(0)^{T} P \bar{x}(0),
\end{aligned}
$$

and then, for $x(0)=\bar{x}(0)$, we have that

$$
V(x(\tau)) \leq \bar{x}(\tau)^{T} P \bar{x}(\tau)=x(0)^{T} e^{-\lambda \tau I_{n}} P e^{-\lambda \tau I_{n}} x(0) .
$$

This means that the trajectories of systems (7) and $\dot{\bar{x}}=-\lambda \bar{x}$ starting at the same point $x(0)$ and after time $\tau$ are such that $V(x(\tau)) \leq V(\bar{x}(\tau))$, for every $\tau \geq 0$. Now we prove that conditions (20) and (21) imply that the value of $V(x)$ decreases between two jumping instant. From the temporal regularization, it is sufficient to prove that the variation (possibly positive) of function $V(x)$ during a jump plus the variation of $V(x)$ after $\rho$ of flowing is non-positive. Such condition must be verified when the state is in the jump set $\mathscr{J}$. Consider a jump at time 0 (no loss of generality is induced) followed by a flowing interval of duration $\rho$. 
Denote with $x_{0}^{-}$the state before the jump and $x_{0}^{+}$the state after the jump. Then, for every $x_{0}^{-} \in \mathscr{J}$, the condition reads

$$
V\left(x_{0}^{+}\right)-V\left(x_{0}^{-}\right)+\int_{0^{+}}^{\rho} \dot{V}(x) d t \leq 0,
$$

and thus, being $V\left(x_{0}^{-}\right)=\left(x_{0}^{-}\right)^{T} P x_{0}^{-}$, we have

$$
x(\rho)^{T} P x(\rho) \leq\left(x_{0}^{-}\right)^{T} P x_{0}^{-} .
$$

Condition (23) means that $V(x)$ before the jump is greater than (or equal to) the value after time $\rho$, when a successive jump could occur. From (22), with $x(0)=x_{0}^{+}$, we have that $\left(x_{0}^{+}\right)^{T} e^{-\lambda \rho I_{n}} P e^{-\lambda \rho I_{n}} x_{0}^{+} \leq\left(x_{0}^{-}\right)^{T} P x_{0}^{-}$and also

$$
\tilde{g}\left(x_{0}^{-}\right)^{T} P \tilde{g}\left(x_{0}^{-}\right)=\left(x_{0}^{+}\right)^{T} P x_{0}^{+} \leq\left(x_{0}^{-}\right)^{T} e^{\lambda \rho I_{n}} P e^{\lambda \rho I_{n}} x_{0}^{-},
$$

are equivalent to condition (23). Finally, since, as shown in the proof of Property 2, we have

$$
\tilde{g}(x)^{T} P \tilde{g}(x) \leq x^{T} \tilde{N}(J(x))^{T} P \tilde{N}(J(x)) x,
$$

for every $x \in \Omega$ and for a proper $J(x) \subseteq \mathbb{N}_{n_{c}}$, (see (19)), then condition (21) implies (24) for every $x \in \Omega \cap \mathscr{I}$ and $\tau \leq \rho$. In fact, posing $x=x_{0}^{-}$and applying the S-procedure, we have

$$
\begin{gathered}
\tilde{g}(x)^{T} P \tilde{g}(x) \leq x^{T} \tilde{N}(J(x))^{T} P \tilde{N}(J(x)) x \leq \\
\leq x^{T} e^{\lambda \rho I_{n}}(P+\sigma M) e^{\lambda \rho I_{n}} x,
\end{gathered}
$$

that leads to satisfaction of condition (24), and hence (23), for every $x \in \Omega$ such that $x \in \mathscr{J}=\left\{x \in \mathbb{R}^{n}: x^{T} M x \leq 0\right\}$.

Remark 4: Function $V(x)$ in Theorem 2 is not necessarily decreasing along the trajectories of system (7), due to jumps, but a decreasing Lyapunov function can be constructed.

\section{Computational issues}

Some computation oriented considerations on how to obtain an ellipsoidal estimation of the basin of attraction for saturated hybrid systems (7) follow. We propose an LMI formulation of the condition provided by Theorem 2 .

Proposition 3: Consider the hybrid system in (7). Suppose that there exist $W \in \mathbb{R}^{n \times n}$ symmetric positive definite, $\lambda>0$, $\hat{Z}(i, J) \in \mathbb{R}^{1 \times n}$ and $\tilde{Z}(j, J) \in \mathbb{R}^{1 \times n}$ for every $I \subseteq \mathbb{N}_{m}$ and $i \in I$, every $J \subseteq \mathbb{N}_{n_{c}}$ and $j \in J$, such that conditions

$$
\begin{gathered}
\left(\hat{A} W+\sum_{i \in \bar{I}} \hat{B}_{(i)} \hat{K}_{i} W+\sum_{i \in I} \hat{B}_{(i)} \hat{Z}(i, I)+\lambda W\right)+\left(W \hat{A}^{T}+\right. \\
\left.+\sum_{i \in \bar{I}} W \hat{K}_{i}^{T} \hat{B}_{(i)}^{T}+\sum_{i \in I} \hat{Z}(i, I)^{T} \hat{B}_{(i)}^{T}+\lambda W\right) \leq 0, \\
{\left[\begin{array}{cc}
W & \left(W \tilde{A}^{T}+\sum_{j \in \bar{J}} W \tilde{K}_{j}^{T} \tilde{B}_{(j)}^{T}+\sum_{j \in J} \tilde{Z}(j, J)^{T} \tilde{B}_{(j)}^{T}\right) E \\
* & W
\end{array}\right] \geq 0,} \\
{\left[\begin{array}{cc}
1 & \hat{Z}(i, I) \\
* & W
\end{array}\right] \geq 0, \forall i \in I, \quad\left[\begin{array}{cc}
1 & \tilde{Z}(j, J) \\
* & W
\end{array}\right] \geq 0, \forall j \in J,}
\end{gathered}
$$

are satisfied for every $I \subseteq \mathbb{N}_{m}$ and $J \subseteq \mathbb{N}_{n_{c}}$, where $E=$ $e^{-\lambda \rho I_{n}}$. Then set $\Omega=\mathscr{E}(P)$, with $P=W^{-1}$, is an ellipsoidal estimation of the basin of attraction and a local Lyapunov function defined in $\Omega$ for the hybrid system in (7) can be determined.
Proof: The proposition stems from Theorem 2. In fact, it can be proved, using standard matrix inequalities manipulation techniques, that (25)-(27) imply the conditions of the theorem, with $W=P^{-1}, \hat{Z}(i, I)=\hat{H}(i, I) W$ and $\tilde{Z}(j, J)=$ $\tilde{H}(j, J) W$, for every $I \subseteq \mathbb{N}_{m}$ and $i \in I$, every $J \subseteq \mathbb{N}_{n_{c}}$ and $j \in J$. The only main difference is that condition (21), which ensures that $V(x)$ is non-increasing between two jumps in $\Omega \cap \mathscr{J}$, is relaxed in (26) imposing the condition over the whole set $\Omega$. Condition (27) assures that $|\hat{H}(i, I) x| \leq 1$ and $|\tilde{H}(j, J) x| \leq 1$, for all $I \subseteq \mathbb{N}_{m}, i \in I, J \subseteq \mathbb{N}_{n_{c}}$ and $j \in J$.

Remark 5: Notice that, as stated in the proof of Proposition 3, the condition on the variation of the value of $V(x)$ during the jump has been imposed over the whole set $\Omega$, although it could have been restricted to the set $\mathscr{J}$. In fact, the term $\sigma M$ in (21), with which we impose the satisfaction of the condition in $\mathscr{J}$, is not present in (26). This, if on one hand could introduce some conservativeness, on the other permits to formulate the problem in LMI form, fixing $\lambda$.

The LMI condition provided in Proposition 3 can be used to pose Problem 1 as an optimization problem. It is left to define the measure of the ellipsoid to be maximized.

Remark 6: We propose here, as an evaluation criterion, to maximize the value of $\beta$ such that the polytope $\beta L=$ $\operatorname{co}\left\{\beta v(k) \in \mathbb{R}^{n}: k \in \mathbb{N}_{V}\right\}$ is contained in the estimate $\Omega=$ $\mathscr{E}(P)$, where $v(k) \in \mathbb{R}^{n}$ with $k \in \mathbb{N}_{V}$ are a set of given points in the state space. The optimization problem results:

$$
\begin{aligned}
& \max _{\beta, \lambda, \tilde{Z}, \hat{Z}, W} \beta \\
& \text { s.t. } \quad(25),(26),(27), \quad \forall I \subseteq \mathbb{N}_{m}, \forall J \subseteq \mathbb{N}_{n_{c}} \text {, } \\
& {\left[\begin{array}{cc}
1 & \beta v(k)^{T} \\
* & W
\end{array}\right] \geq 0, \quad \forall k \in \mathbb{N}_{V},}
\end{aligned}
$$

where, for sake of notational compactness, we denoted with $\tilde{Z}$ and $\hat{Z}$ the matrices $\tilde{Z}(i, I)$ and $\hat{Z}(j, J)$ for every $I \subseteq \mathbb{N}_{m}$ and $i \in I$, every $J \subseteq \mathbb{N}_{n_{c}}$ and $j \in J$. The first set of constraints in (28) ensures that $\mathscr{E}\left(W^{-1}\right)$ is an estimation of the domain of attraction for system (7), and the second one imposes that $\beta v(k) \in \mathscr{E}\left(W^{-1}\right)$, for every $k \in \mathbb{N}_{V}$.

Finally notice that, although the constraints (25) and (26) are not linear in the optimization variables, they are LMI for fixed value of $\lambda$. Then, in practice, the problem can be solved for different values of $\lambda>0$, to obtain an estimation of the maximal value of $\beta$, and then the maximal estimation of the domain of attraction.

\section{NUMERICAL EXAMPLE: RESET SYSTEM}

We consider the system proposed in [7] characterized by the linear open-loop one-dimensional unstable system:

$$
\left\{\begin{array}{l}
\dot{x}_{p}(t)=0.1 x_{p}(t)+\varphi\left(y_{c}(t, j)\right) \\
y_{p}(t)=x_{p}(t)
\end{array}\right.
$$

with $p=n_{p}=m=1$, and the stabilizing PI controller

$$
\left\{\begin{array}{l}
\dot{x}_{c}(t, j)=-0.2 y_{p}(t) \\
y_{c}(t, j)=x_{c}(t, j)-2 y_{p}(t),
\end{array}\right.
$$

with $n_{c}=1$, and discrete-time dynamics characterizing the reset behavior with saturation is

$$
x_{c}(t, j+1)=x_{c}(t, j)+\varphi\left(-x_{c}(t, j)\right) .
$$


The temporal regularization parameter is $\rho=2$.

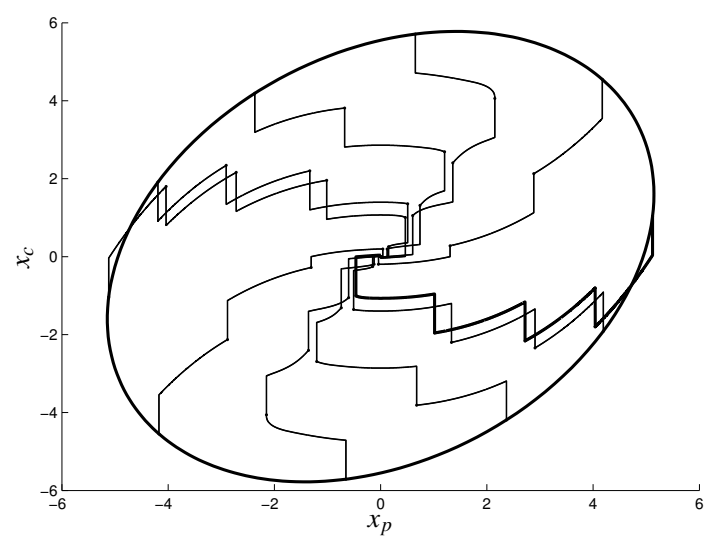

Fig. 1. Set $\Omega$ and trajectories of the reset system.

We solve the optimization problem (28) where points $v(k)$, with $k \in \mathbb{N}_{4}$, are the vertices of the square set $L=\left\{x \in \mathbb{R}^{2}\right.$ : $\left.\|x\|_{\infty} \leq 1\right\}$, and for different values of $\lambda$. We found that the value of $\lambda=0.02$ provides the best value (among those tested) of $\beta$, that is $\beta=3.2689$ with

$$
P=\left[\begin{array}{cc}
0.0409 & -0.0101 \\
* & 0.03241
\end{array}\right] \text {. }
$$

The ellipsoid $\Omega=\mathscr{E}(P)$ is an estimation of the domain of attraction of the reset system, regardless on the region of jump. This can be noticed in Figure 1, where the ellipsoid is depicted with some trajectories of the reset system assuming that the jump can occur at any point of $\Omega$. In particular, the trajectory marked in bold line with initial condition $x(0)=x_{0}^{-}=[5.1188,1.0376]^{T}$ is interesting. Notice that with the first jump at time 0 the trajectory leaves the ellipsoid, then the value of $V(x)$ increases, i.e. $V\left(x_{0}^{+}\right)>1$. At the time of the second jump the state is contained in the ellipsoid, which means that $V(x(\rho))<1$. Then $V(x)$ decreases between the two jumps, as ensured by Proposition 3. The value of $V(x)$ along the trajectory starting at $x=[5.1188,1.0376]^{T}$ is shown Figure 2, in thick continuous line. Function $V(x)$ passes at time $t=0$ from the value of 1 to 1.0686 , but at the time of the second jump, $t=2$, its value is smaller than 1 , in particular $V(x(2))=0.9196$. The dashed thin line in Figure 2 is the evolution of $V(x)$ of the trajectory of the saturated system starting in $x=[5.1188,1.0376]^{T}$, in absence of the discrete-time dynamics, that is with $\mathscr{J}=\emptyset$. It can be noticed that, although at first the reset behavior leads to higher values of $V(x)$, the effect of the reset is beneficial accelerating the convergence of $V(x)$ to zero.

\section{CONCLUSIONS}

In this paper we dealt with the problem of characterizing and computing an ellipsoidal estimation of the basin of attraction for saturated hybrid systems. The results presented are based on a geometrical approach to the analysis of saturated functions. The proposed approach permits to recover or extend some results present in literature for continuous-time

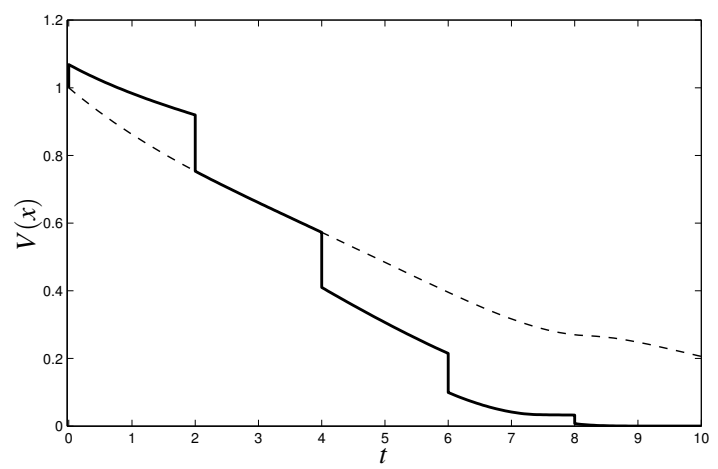

Fig. 2. Function $V(x)$ along trajectory starting at $x=\left[\begin{array}{lll}5.1188 & 1.0376\end{array}\right]^{T}$.

and discrete-time saturated systems, as well as to characterize local convergence for saturated hybrid systems.

\section{REFERENCES}

[1] T. Alamo, A. Cepeda, M. Fiacchini, and E. F. Camacho. Convex invariant sets for discrete-time Lur'e systems. Automatica, 45:10661071, 2009.

[2] T. Alamo, A. Cepeda, and D. Limon. Improved computation of ellipsoidal invariant sets for saturated control systems. In Proc. 44th IEEE Conf. on Decision and Control and European Control Conference CDC-ECC 2005, pages 6216-6221, Dec. 2005.

[3] T. Alamo, A. Cepeda, D. Limon, and E. F. Camacho. A new concept of invariance for saturated systems. Automatica, 42:1515-1521, 2006

[4] M. S. Branicky, V. S. Borkar, and S. K. Mitter. A unified framework for hybrid control: model and optimal control theory. IEEE Transactions on Automatic Control, 43:31-45, 1998.

[5] R. Goebel, J. Hespanha, A.R. Teel, C. Cai, and R. Sanfelice. Hybrid Systems: Generalized Solutions and Robust Stability. Proc. IFAC: Symp on Nonlinear Control Systems, Stuttgart, Germany, pages 1-12, 2004.

[6] J. M. Gomes Da Silva Jr. and S. Tarbouriech. Local stabilization of discrete-time linear systems with saturating controls: An LMI-based approach. IEEE Transactions on automatic control, 46:119-125, 2001.

[7] J. M. Gomes da Silva Jr. and S. Tarbouriech. Antiwindup design with guaranteed regions of stability: an LMI-based approach. IEEE Transactions on Automatic Control, 50(1):106-111, 2005.

[8] R. L. Grossmann, A. Nerode, A. P. Ravn, and H. Rischel. Hybrid systems. Lecture Notes in Computer Science, 736, 1993.

[9] T. Hu and Z. Lin. Exact characterization of invariant ellipsoids for single input linear systems subject to actuator saturation. IEEE Transactions on Automatic Control, 47(1):164-169, jan. 2002.

[10] T. Hu, Z. Lin, and B. M. Chen. Analysis and design for discretetime linear systems subject to actuator saturation. Systems \& Control Letters, 45(2):97-112, 2002.

[11] D. Liberzon. Switching in Systems and Control. Boston: Birkhauser, 2003.

[12] T. Loquen, S. Tarbouriech, and C. Prieur. Stability analysis for reset systems with input saturation. In Proc. 46th IEEE Conf. on Decision and Control, pages 3272-3277, New Orleans, LA, USA, 2007.

[13] D. Nesic̀, L. Zaccarian, and A.R. Teel. Stability properties of reset systems. Automatica, 44(8):2019-2026, 2008.

[14] R. T. Rockafellar. Convex Analysis. Princeton University Press, USA, 1970.

[15] R. Schneider. Convex bodies: The Brunn-Minkowski theory, volume 44. Cambridge University Press, Cambridge, England, 1993.

[16] Z. Sun and S. S. Ge. Switched Linear Systems: Control and Design. London: Springer-Verlag, 2005.

[17] S. Tarbouriech, C. Prieur, and J.M. Gomes da Silva Jr. Stability analysis and stabilization of systems presenting nested saturations. IEEE Transactions on Automatic Control, 51(8):1364-1371, 2006.

[18] L. Zaccarian, D. Nesić, and A.R. Teel. First order reset elements and the Clegg integrator revisited. In Proc. 2005 American Control Conference, pages 563-568, Portland, OR, USA, June 2005. 